\title{
Aeroacoustic Façade Noise Validation: A Comparison of CFD and Wind Tunnel Tests
}

\author{
Nathaniel L. Jones and Alexej Goehring \\ Arup, San Francisco, USA
}

\begin{abstract}
Computational fluid dynamics (CFD) analysis is a valuable alternative to wind tunnel testing for analysing and predicting aeroacoustic noise. However, transient simulations require a very fine grid and short time step to resolve acoustic pressure fluctuations, resulting in a computationally intensive process. This paper investigates the possibility of using steady state CFD simulations as a fast alternative to transient simulations for characterizing the noise production of perforated façade panels. It compares both transient and steady-state Reynolds-averaged Navier-Stokes (RANS) simulations of aeroacoustically active perforated panels to measurements of specimens in a reverberation chamber. Accurate sound power predictions can be achieved for broadband noise from normally incident wind on perforated panels, but sound power and frequency remain difficult to predict for oblique wind directions.
\end{abstract}

\section{Introduction}

Aeroacoustic noise is unwanted sound generated when turbulent air passes over an object, stimulating audible frequencies. Recently, aeroacoustic noise has become a subject of concern for buildings, where façade elements such as screens and shading devices may create windinduced noise (Hamer, 2006; Lisboa, Ottieri, \& González, 2015). Perforated screens, which provide shade and privacy while allowing natural ventilation and preserving views to the outdoors, are especially vulnerable to windinduced whistling. Recordings from wind tunnel testing can reveal aeroacoustic phenomena (Feng, 2012; Blinet, Kerdudou, Chéné, \& Jacqus, 2015), but such tests are expensive, making them infeasible in early design. The ability to predict noise generation quickly using numerical methods will help designers select suitable façade elements for wind-prone areas.

In this paper, we compare sound measurements from earlier wind tunnel experiments to computational fluid dynamics (CFD) modelling of aeroacoustic noise. The sound of air passing through perforated panels was recorded in a reverberation chamber and characterized according to sound power in 1/3-octave bands. We reproduced several of these tests using Reynoldsaveraged Navier-Stokes (RANS) computational models and performed sensitivity analysis: First, we tested the effect of CFD mesh resolution on the sound power level predicted by steady state simulation. Second, we tested for dependence of sound power on perforation size. Third, we investigated the ability of RANS simulation to model sound power dependence on wind incidence angle. Fourth, we performed similar analysis for variation in wind speed. Finally, we performed transient RANS simulations to detect the dominant frequencies of air vibration and compare those to recorded tonal noise. Using this data, we hope to predict real-world aeroacoustic noise characteristics for risk assessments based on relatively accessible simulation methods.

\section{Background}

While the whistling and humming noises of wind on buildings are familiar to many, the mechanisms that generate this noise are often unclear. Several phenomena can be responsible: Aeroacoustic noise may be generated by organized wakes (Kármán vortex streets) trailing downwind of rods, tubes, or perforations. Tubes and cavities may also act as Helmhotz resonators. Pressure fluctuations in air may excite structural vibrations as well; this can produce resonance when the frequency corresponds to an eigenmode of structural vibration.

Aeroacoustic noise is problematic when it includes either a load broadband component or a clear tone in the range of human hearing. Human beings hear frequencies between 20 and $20,000 \mathrm{~Hz}$, but we are most sensitive to frequencies in the range of speech $(500-5000 \mathrm{~Hz})$ (Figure 1). Additionally, tonal sources-those that include a dominant frequency-will be heard more clearly than broadband noise and pose a greater nuisance.

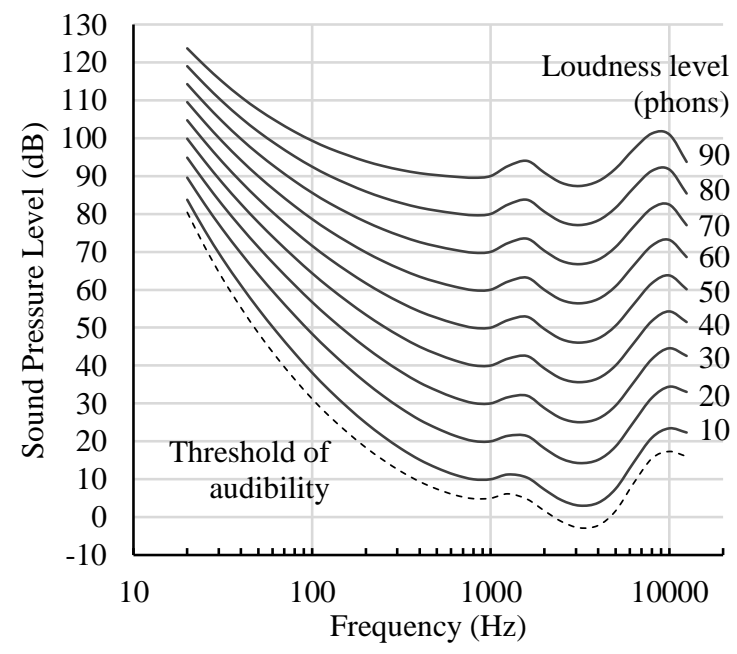

Figure 1: Curves of equal loudness for frequencies in the range of human hearing (after ISO, 2003). 


\section{Frequency}

For Kármán vortex streets, the frequency $f$ at which eddies shed from the structural element is related to the dimensionless Strouhal number St:

$$
S t=\frac{f \times D}{U}
$$

where $U$ is the incident wind speed and $D$ is the characteristic length, typically the diameter of the structural element. Kármán vortex streets develop in the transition from laminar to turbulent flow, where the Strouhal number is around 0.2 .

For groupings of multiple elements, interaction between wakes changes the tonal characteristics. Parallel bars produce higher Strouhal numbers $(\sim 0.3)$ when placed close together, although the observed sound is mainly broadband (Blinet, Kerdudou, Chéné, \& Jacqus, 2015). For perforated panels, a modified characteristic dimension $t^{*}$ is proposed to preserve the Strouhal number's value of 0.2 :

$$
t^{*}=\frac{\varphi}{0.01} t
$$

where $t$ is the panel thickness and $\varphi$ is the hole diameter in meters (Feng, 2012). This does not account for hole spacing, which might also affect characteristics of the wake.

\section{Sound Power}

Noise levels can be approximated by rearrangement of the Navier-Stokes equations. Lighthill (1952) proposed an analogy that reformulates the Navier-Stokes equation as a wave equation with source terms. Considering turbuence as the only sound source, Lighthill's analogy reduces to the Proudman Broadband Noise model (Proudman, 1952), which relates acoustic power $P$ in $\mathrm{W} / \mathrm{m}^{3}$ to the density of air $\rho$, eddy dissipation $\varepsilon$, turbulence kinetic energy $k$, speed of sound $c$, and a constant $(\alpha=0.1)$ :

$$
P=\alpha \rho \varepsilon\left(\frac{\sqrt{2 k}}{c}\right)^{5}
$$

The total sound power $P_{V}$ in watts generated in a volume $V$ is found by taking the volume integral:

$$
P_{V}=\int_{V} P d V
$$

The Proudman model can be applied to a steady state RANS simulation, which solves for $k$ and $\varepsilon$ at the subgrid scale. This technique has previously been used to model the noise of gas jets (ANSYS, 2015), automobiles (Ringwall, 2017), and façade panels (Jones \& Goehring, 2018).

Wind tunnel tests are frequently used to measure aeroacoustic noise levels. Sound may be recorded in an anechoic wind tunnel (Iglesias, Thompson, \& Smith, 2016) or in a reverberation chamber through which air is forced (Feng, 2012). Frequency and amplitude of structural vibrations in a wind tunnel may also be recorded with high speed cameras (Purohit, Darpe, \& Singh, 2016). However, even when designed for acoustic testing, measurements in similarly constructed wind tunnels may differ by $10-15 \mathrm{~dB}$ (Devenport, et al., 2013). It is therefore preferable to compare test samples to reference samples with known sound power.

Several studies have compared wind tunnel recordings to aeroacoustic CFD simulations. A comparison of Large Eddy Simulation (LES) and Detached Eddy Simulation (DES) found LES a closer match to wind tunnel measurements, though both simulations produced errors in some frequency bands upwards of $10 \mathrm{~dB}$ (Ask, 2006). Using a Lattice Boltzmann method, transient pressure variations in a resonant cavity can be observed directly, although the amplitude is higher than that observed in wind tunnels (de Jong, Bijl, Hazir, \& Wiedemann, 2013). These transient methods are useful for their ability to simulate sound pressure and frequency in addition to overall sound power, but they requre small time steps and fine mesh resolutons, making them computationally expensive.

\section{Experiments}

Our study is based on previous wind tunnel tests in which Feng (2012) measured tonal noise generated by perforated plates in a flow field. The test environment consisted of a pressurized anechoic chamber connected by an opening to a reverberating chamber (Figure 2). A perforated panel measuring $400 \mathrm{~mm} \times 400 \mathrm{~mm}$ with a thickness of $1.5 \mathrm{~mm}$ was placed in the opening between the two chambers at various rotations angles. Feng measured sound power levels using a rotating microphone in the reverberation chamber in accordance with international standard EN ISO 3741:2010.

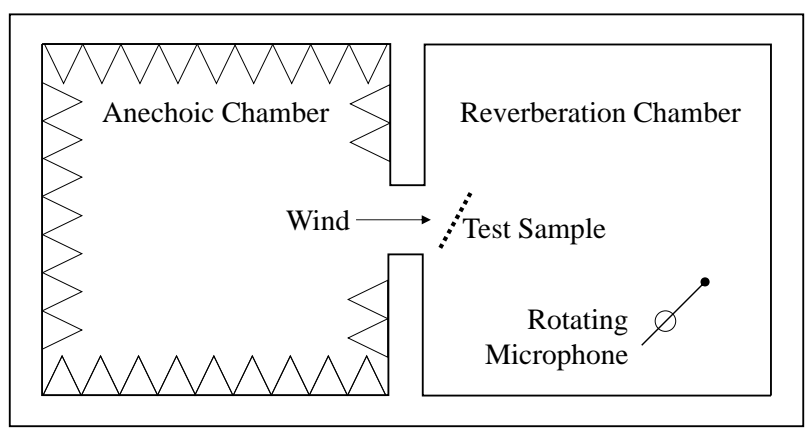

Figure 2: The test chamber used by Feng (2012) consisted of a anechoic chamber in constant pressurization connected to a reverberation chamber.

We duplicated several of these tests in CFD simulations using ANSYS CFX (2018). Although the exact geometry of the panels is not recorded, we estimate the perforation openness and total number of holes per panel based on documented perforation sizes and spacing for the four panels listed in Table 1. The number of perforations we estimate accounts for holes obstructed by the $25-\mathrm{mm} \mathrm{L}$ shaped supports. We ignore Feng's sample 1 as this panel had an irregular hole pattern.

Feng observed broadband aeroacoustic noise for wind with normal incidence on the panels and tonal characteristics when panels were oriented at $60^{\circ}$ or more to oncoming wind. We consider two geometries in our CFD simulations: First, we perform sensitivity analysis 
on mesh resolution and hole size with a panel oriented normal to the wind direction. Second, we test the effect of wind speed on sound power and frequency with a panel oriented at $75^{\circ}$ to the wind, which produced the most distinct tones in physical tests. For the normal orientation, we model a $200 \mathrm{~mm} \times 200 \mathrm{~mm} \times 200 \mathrm{~mm}$ domain with symmetry conditions on the top and side to represent the entire panel. For arbitrary wind incidence angles, we eliminate the vertical symmetry condition and expand the domain to include the entire $400-\mathrm{mm}$ panel width. For the $75^{\circ}$ orientation where we introduced transient simulation, we model the entire width of the panel and introduce translational periodic boundaries to account for its full height.

Table 1: Panel perforation data.

\begin{tabular}{|c|c|c|c|c|c|}
\hline \multirow{2}{*}{ Sample } & \multirow{2}{*}{$\begin{array}{c}\text { Diam. } \\
(\mathbf{m m})\end{array}$} & \multicolumn{2}{|c|}{ Spacing (mm) } & Open & Hole \\
\cline { 3 - 4 } & $\mathbf{x}$ & $\mathbf{y}$ & $\mathbf{\%}$ & Count \\
\hline 2 & 12 & 27 & 17.5 & 23.9 & 286 \\
\hline 3 & 8 & 18 & 11.6 & 24.1 & 627 \\
\hline 4 & 11 & 23 & 15 & 27.5 & 390 \\
\hline 5 & 10 & 21 & 13.5 & 27.7 & 448 \\
\hline
\end{tabular}

\section{Mesh Resolution}

Because the Proudman model depends on eddy dissipation and turbulence kinetic energy calculated at subgrid scale, sufficient mesh resolution is necessary to localize areas of sound production. Figure 3 demonstrates that a coarse CFD mesh will underestimate the total sound power production, which occurs mostly in the areas shaded red. However, finer mesh resolution leads to increased simulation time. For practical considerations, our goal is to use the coarsest mesh that still resolves areas of sound production.
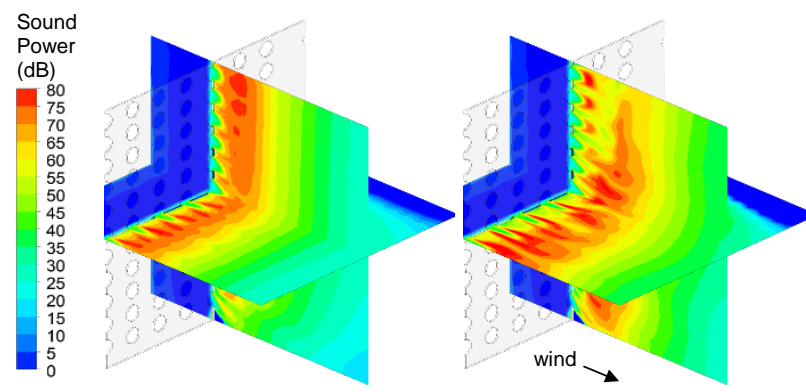

Figure 3: The coarse mesh with a maximum element size of $10 \mathrm{~mm}$ near the panel (left) does not resolve local turbulence as well as the fine mesh with a maximum element size of $1 \mathrm{~mm}$ near the panel (right).

For sample 2 (12-mm hole diameter), we first ran a CFD simulation on a uniform 1-mm cubic mesh to determine the region in which sound production occurs. Under a 12 $\mathrm{m} / \mathrm{s}$ wind, this region extends $50 \mathrm{~mm}$ downwind from the panel. We then tested several tetrahedral meshes with varying mesh densities around the production region (Figure 4). Capping the cell edge length in this region at $3 \mathrm{~mm}$ or smaller produced results within $3 \mathrm{~dB}$ of the 54.5 $\mathrm{dB}$ broadband signal recorded by Feng after subtracting background noise.

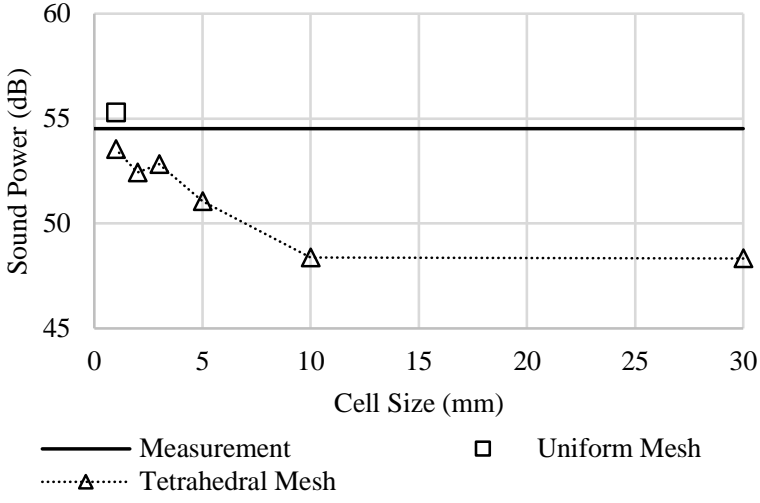

Figure 4: RANS simulations with mesh resolution smaller than $3 \mathrm{~mm}$ predicted sound power levels within

$3 \mathrm{~dB}$ for normal incidence to a perforated panel.

\section{Hole Size}

While frequency is generally understood to vary with perforation size according to the Strouhal number, less is known about variation in sound power. We modelled the remaining samples $(3,4$, and 5) under normal incidence conditions with a 2-mm cap on mesh cell edge length in the sound production region (Figure 5) and compared the sound power according to the Proudman model with that measured by Feng (Figure 6). In simulation, we observe a slight decrease in sound power as hole size increases, although due to the varying number of holes per panel, this represents an increase in sound power per hole. The measured sound powers do not show a clear trend with respect to hole size, although some dependence is apparent with respect to perforation openness (Figure 7). Nearly all differences are within the error margin established in the previous test.

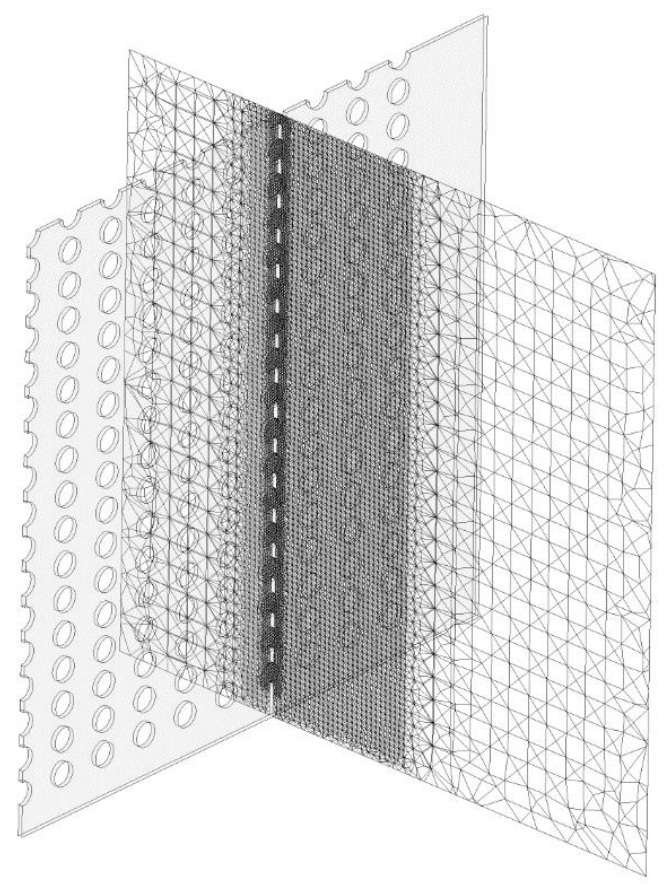

Figure 5: The chosen mesh includes a dense region with a maximum cell edge length of $2 \mathrm{~mm}$ in the sound production region up to $50 \mathrm{~mm}$ downwind of the panel. 


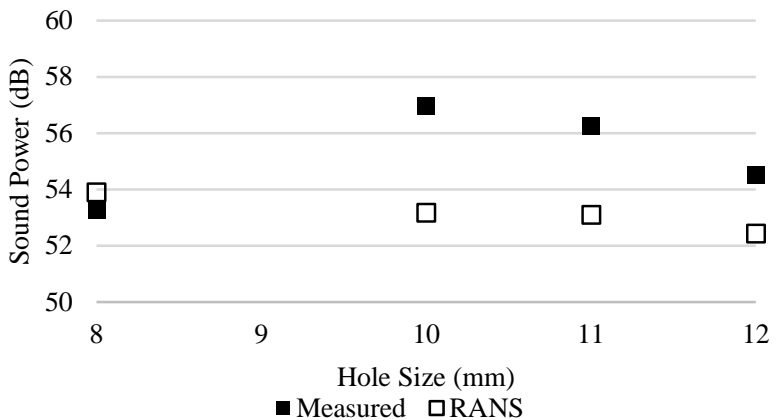

Figure 6: Simulation shows a slight dependence of sound power on hole size, which is not seen in wind tunnel test data.

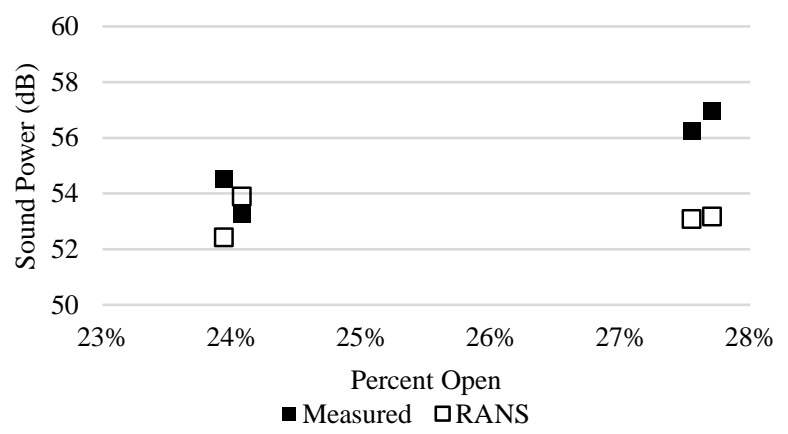

Figure 7: In wind tunnel tests, the openness of the perforation pattern affected sound power regardless of holes size.

\section{Wind Incidence Angle}

In Feng's experiment, the panel's angle with respect to the direction of air movement affected the sound output. Tonal noise occurred for wind at $60^{\circ}$ and $75^{\circ}$ angles to the panel's normal direction, while broadband noise occurred at all angles. We investigated the effect of wind incidence angle on sound power for sample 2. For the angled panel, we cannot model the domain using a vertical symmetry plane because air is likely to be diverted along the panel as well as flow through it. Instead, we double the width of the domain to include the entire panel (Figure 8).

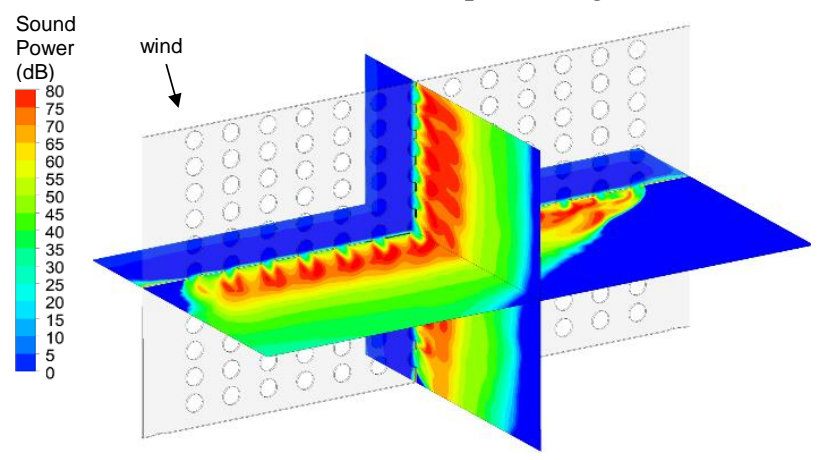

Figure 8: When wind blows at an angle to the panel, air moves along the panel as well as through the holes.

Sound production of sample 2 under $12 \mathrm{~m} / \mathrm{s}$ wind at $30^{\circ}$ orientation is shown.

Although the Proudman model's prediction closely matches the observed sound power for normal incidence, it diverges greatly at steep angles (Figure 9). Feng observed peak sound power generation for wind at $75^{\circ}$ to the panel's normal direction, which could be up to $15 \mathrm{~dB}$ louder than wind at normal incidence. This indicates that other acoustic mechanisms apply to the angled panel in addition to aeroacoustic production from vorticity included in the Proudman model. Because the space behind the panel is open and should not act as a Helmholtz resonator, we surmise that wind induces mechanical vibrations in the panel to account for the missing sound power. This assumption is supported by wind tunnel observations in which the sound production of a perforated panel can be stopped by placing a finger on the panel (Wood, 2018).

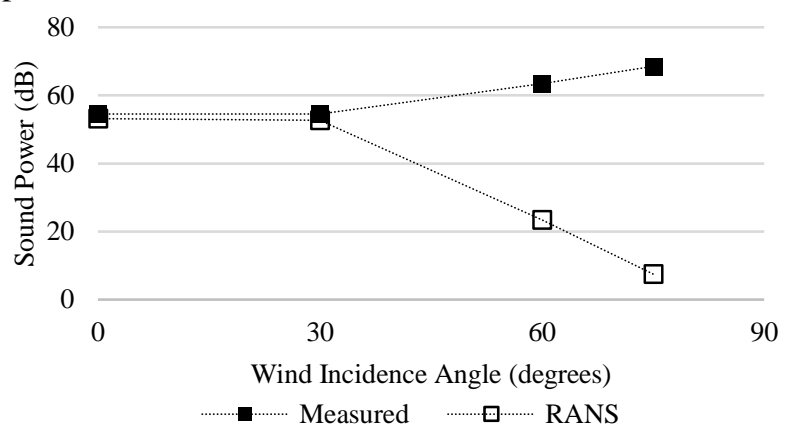

Figure 9: Measured and simulated sound power match closely at normal incidence but have different characteristics for extreme wind angles.

\section{Wind Speed}

We investigated the effect of wind speed on sound power for sample 2 oriented at $75^{\circ}$ to the wind. Given the steep angle, the simulation domain extends beyond the edge of the panel, allowing air to flow around the panel on both sides. It is also necessary to include the L-shaped supports in the model for their shielding effect (Figure 10). Indeed, without the supports in place, wind flowing around the panel reduces the size of the sound-producing turbulent region downstream of the holes and consequently also the sound power. Again, we apply a higher mesh density to the sound production region downstream of the panel between the L-shaped supports.

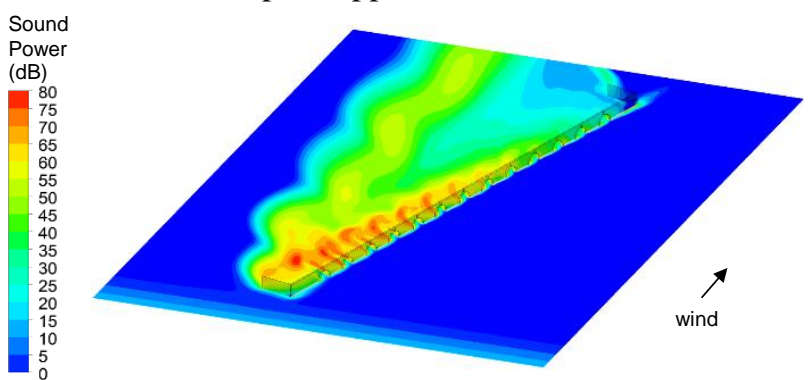

Figure 10: The L-shaped supports shield a region

behind the angled panel in which sound production occurs. Sound production of sample 2 under $15 \mathrm{~m} / \mathrm{s}$ wind at $75^{\circ}$ orientation is shown.

Sound power increases with wind speed in both measurements and simulation. Although the measured sound power remains higher than the simulation values for the $75^{\circ}$ orientation as in the previous test, both the recording and simulation show similar gains with respect to $12 \mathrm{~m} / \mathrm{s}$ wind as wind speed changes (Figure 11). Deviation between the gains for measured and simulated results is only apparent at the lowest wind speed. 


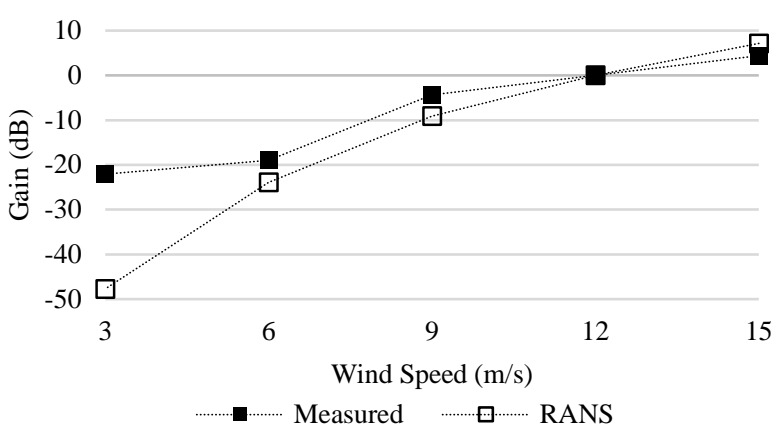

Figure 11: Sound power increases with wind speed for simulations of a screen with $75^{\circ}$ incidence angle, as shown by the gain relative to $12 \mathrm{~m} / \mathrm{s}$ wind for both measured and simulated sound power.

\section{Frequency}

Feng observed panels oriented at $75^{\circ}$ incidence to produce just-audible tones. If this tonal signal results from resonant vibrations in the panel itself, then these vibrations should be observable in transient CFD simulation. We carried out transient simulations on a $2.5 \times 10^{-5}$-s time step and monitored the force vector applied by the wind on various patches of the panel. After applying a fast Fourier transform, we compare the panel's dominant frequency in our transient RANS simulations to measured frequencies and to predicted frequencies assuming a Strouhal number of 0.2 (Figure 12). While the Strouhal prediction aligns closely with observations for lower wind speeds, the RANS simulations trend in the opposite direction.

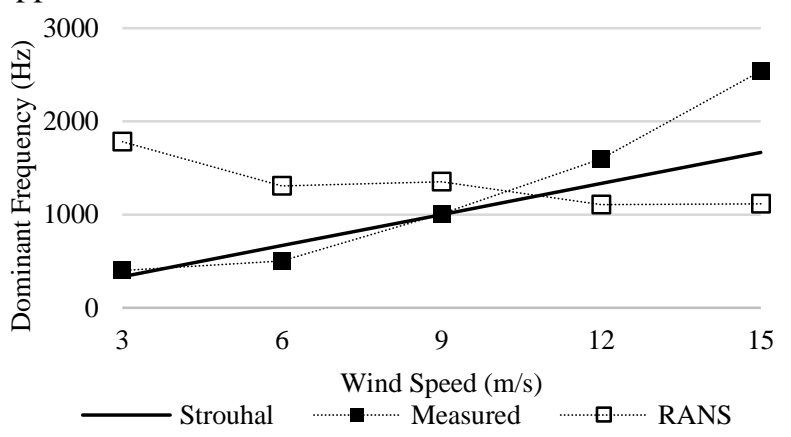

Figure 12: Measured tonal frequencies increase as predicted by the Strouhal number, but simulations of a screen with $75^{\circ}$ incidence do not show this trend.

\section{Discussion}

We set out to investigate whether fast steady state CFD simulations with coarse meshes could predict characteristics of aeroacoustic noise. For wind with normal incidence, the Proudman model produces a reasonably accurate prediction of aeroacoustic sound power based on steady state RANS simulation. A coarse mesh can generally be used except in the region where aeroacousic noise is generated, which requires a fine mesh density. Using tetrahedral meshes with maximum cell edge length of $2 \mathrm{~mm}$ in the regions $50 \mathrm{~mm}$ downstream of the panel, we achieved sound power predictions within 4 $\mathrm{dB}$ of measured values for $8-\mathrm{mm}$ to $12-\mathrm{mm}$ diameter holes. This accuracy roughly matches the $3-5 \mathrm{dBA}$ sound power level variation necessary to achieve a perceptible difference for human listeners in environments with background noise (Liu, 2012), and is therefore reasonable for early design analysis.

For wind obliquely incident on panels, the Proudman model underestimates sound power observed by Feng (2012). Given the tonal behaviour observed in panels oriented $75^{\circ}$ to the wind, this may reflect the fact that the Proudman model does not account for some mechanisms of tonal noise production, such as monopole and dipole sources, interference patterns in Kármán vortex streets, and induced vibrations in the panel. Interactions at scales smaller than the 2-mm grid in our simulation may also play a role for the angled panel, as the panel's thickness dimension becomes more critical. Observations of aeroacoustic differences caused by panel manufacturing methods support this explanation (Wood, 2018). Our method of assuming translational periodicity for transient simulations may also factor into this, as we do not account for potential wake interactions in the vertical axis or for acoustic effects at the top and bottom edges of the panel. Follow-up work should simulate eigenmode vibrations of panels, including effects of perforations, to determine whether structural resonance plays a role in the oblique panels' acoustic behaviour. New wind tunnel tests that observe or isolate specific noise production mechanisms will also be useful to develop a reliable model for noise generation from angled panels. This is critical for understanding aeroacoustic behaviour of building façades, as local air currents most commonly occur parallel to the façade (Jones \& Goehring, 2018).

For aeroacoustic noise risk assessment using RANS simulation, sound power levels from normally incident wind should be used to represent a panel's acoustic behaviour. This case offers the best correspondence with measured values and is within $15 \mathrm{~dB}$ of the maximum sound power level in the recorded cases we analysed. The maximum aeroacoustic production of a panel can therefore be estimated at $15 \mathrm{~dB}$ higher than the simulated sound power at normal incidence. Dominant acoustic frequency can be estimated from the Strouhal number. We caution that this method does not predict the occurrence of tonal noise; rather, it predicts what tone will be emitted if tonal noise occurs.

\section{Conclusion}

We reproduced testing conditions in RANS simulation for several aeroacoustically active perforated panels that have previously been subjected to laboratory tests in the reverberating chamber of a wind tunnel. Steady state RANS models are advantageous because they run in minutes, compared to days for transient models. For wind at normal incidence angles, these models can predict aeroacoustic sound power with reasonable accuracy. In our tests, we could predict sound power within $4 \mathrm{~dB}$ for a variety of perforation hole sizes. However, for wind incident on the panel at an angle, measured sound power may be higher than predicted by the Proudman model. A reasonable estimate for maximum sound power at any wind angle is $15 \mathrm{~dB}$ higher that the Proudman model prediction at normal incidence. Further work is needed to 
understand the aeroacoustic mechanisms involved in perforated panels.

\section{Acknowledgement}

The authors offer their sincere appreciation to Graeme Wood and Steve Downie for their advice and support. This work was funded by Invest in Arup project 18911.

\section{References}

\section{ANSYS. (2018). CFX 19.1.}

ANSYS, Inc. (2015). Summary: Modeling acoustics of a coaxial jet using broadband noise model. Cheyenne (USA): SAS IP, Inc.

Ask, J. (2006). The sub-critical flow past a generic side mirror and its impact on sound generation and propagation. 12th AIAA/CEAS Aeroacoustics Conference, (pp. 1-20). 8-10 May 2006, Cambridge (USA).

Blinet, T., Kerdudou, P., Chéné, J.-B., \& Jacqus, G. (2015). Wind noise generated by facade elements on buildings: A simple measurement method and case studies. Internoise 2015. San Francisco (USA).

de Jong, A. T., Bijl, H., Hazir, A., \& Wiedemann, J. (2013). Aeroacoustic simulation of slender partially covered cavities using a Lattice Boltzmann method. Journal of Sound and Vibration, 332(7), 1687-1703. doi:10.1016/j.jsv.2012.09.040

Devenport, W. J., Burdisso, R. A., Borgoltz, A., Ravetta, P. A., Barone, M. F., Brown, K. A., \& Morton, M. A. (2013). The Kevlar-walled anechoic wind tunnel. Journal of Sound and Vibration, 332(17), 3971-3991. doi:10.1016/j.jsv.2013.02.043

Feng, L. (2012). Tone-like signal in the wind-induced noise of perforated plates. Acta Acustica united with Acustica, 98(1), 188-194. doi:10.3813/AAA.918504

Hamer, M. (2006, August 2). Buildings that whistle in the wind. New Scientist. Retrieved from https://www.newscientist.com/article/mg19125631.3 00-buildings-that-whistle-in-the-wind/

Iglesias, E. L., Thompson, D. J., \& Smith, M. G. (2016). Experimental study of the aerodynamic noise radiated by cylinders with different cross-sections and yaw angles. Journal of Sound and Vibration, 361, 108-129. doi:10.1016/j.jsv.2015.09.044
ISO. (2003). Acoustics-Normal equal-loudness-level contours. ISO 226:2003. Geneva (Switzerland): International Organization for Standardization.

Jones, N. L., \& Goehring, A. (2018). Aeroacoustic facade noise: Predicting wind-induced noise from perforated facade panels. 2018 Building Performance Analysis Conference and SimBuild co-organized by ASHRAE and IBPSA-USA, (pp. 274-281). 26-28 September 2018, Chicago (USA).

Lighthill, M. J. (1952). On sound generated aerodynamically I. General Theory. Proceedings of the Royal Society. Series A, Mathematical and Physical, 211(107), 564-587. doi:10.1098/rspa.1952.0060

Lisboa, M. R., Ottieri, J. C., \& González, A. E. (2015). Noise annoyance due to wind flow interaction with a building's facade. Open Journal of Acoustics, 5, 1-10. doi:10.4236/oja.2015.51001

Liu, W. F. (2012). Comparing sound measurements in the single-family room with open-unit design neonatal intensive care unit: The impact of equipment noise. Journal of Perinatology, 32, 369-373. doi:10.1038/jp.2011.103

Proudman, I. (1952). The generation of noise by isotropic turbulence. Proceedings of the Royal Society. Series A, Mathematical and Physical, 214(1116), 119-132. doi:10.1098/rspa.1952.0154

Purohit, A., Darpe, A. K., \& Singh, S. P. (2016). Experimental investigations on flow induced vibration of an externally excited flexible plate. Journal of Sound and Vibration, 371, 237-251. doi:10.1016/j.jsv.2016.02.039

Ringwall, E. (2017). Aeroacoustic sound sources around the wheels of a passenger car-A Computational Fluid Dynamics study using steady state models to evaluate main sources of flow noise. Master's thesis: Chalmers University of Technology. Retrieved from http://studentarbeten.chalmers.se/publication/250505 -aeroacoustic-sound-sources-around-the-wheels-of-apassenger-car-a-computational-fluid-dynamics-study

Wood, G. (2018). Personal correspondence. 